\title{
Torticollis-keloids-cryptorchidism-renal dysplasia syndrome
}

INSERM

\section{Source}

INSERM. (1999). Orphanet: an online rare disease and orphan drug data base. Torticolliskeloids-cryptorchidism-renal dysplasia syndrome. ORPHA:3341

Torticollis-keloids-cryptorchidism-renal dysplasia syndrome is an extremely rare developmental defect during embryogenesis malformation syndrome characterized by congenital muscular torticollis associated with skin anomalies (such as multiple keloids, pigmented nevi, epithelioma), urogenital malformations (including cryptorchidism and hypospadias) and renal dysplasia (e.g. chronic pyelonephritis, renal atrophy). Additional reported features include varicose veins, intellectual disability and musculoskeletal anomalies. 